Artículo científico

Volumen 31(1):117-128. Enero-abril, 2020

e-ISSN 2215-3608, doi:10.15517/am.v31i1.36815

http://www.revistas.ucr.ac.cr/index.php/agromeso

\title{
Efecto de dosis de nitrógeno en la agronomía y fisiología de plantas de maracuyá ${ }^{1}$
}

\section{Effect of doses of nitrogen in the agronomy and physiology of yellow passion fruit}

\author{
Gustavo Adolfo Rodríguez-Yzquierdo², Héctor Eduardo Pradenas-Aguila ${ }^{3}$, Carmen Amalia Basso-de-Figuera ${ }^{3}$, \\ Marta Barrios-García ${ }^{3}$, Rommel Igor León-Pacheco ${ }^{2}$, Mercedes Pérez-Macias ${ }^{3,4}$
}

1 Recepción: 8 de abril, 2019. Aceptación: 2 de julio, 2019. Este trabajo formó parte del proyecto No 2013-002353 "Ecofisiología del estrés en el cultivo de parchita maracuyá como alternativa tecnológica frente a las principales limitantes en zonas del centro del país" desarrollado en la Universidad Central de Venezuela, Facultad de Agronomía, Venezuela, financiado por el Fondo Nacional de Ciencia y Tecnología (FONACIT).

2 Corporación Colombiana de Investigación Agropecuaria (Agrosavia). Mosquera, Cundinamarca, Colombia. Apdo 344300. grodriguezy@ agrosavia.co (https://orcid.org/0000-0003-3709-8534), rleon@agrosavia.co (autor para correspondencia, https://orcid.org/0000-0002-9928-5282).

3 Universidad Central de Venezuela, Facultad de Agronomía. Maracay, Venezuela. Apdo 2101. hectorpradenas_@hotmail.com, carmen. basso@ucv.ve, marta.barrios@ucv.ve, mercedes.perez@ucv.ve

4 Instituto Nacional de Investigaciones Agrícolas - Centro Nacional de Investigaciones Agropecuarias (INIA-CENIAP). Maracay, Venezuela. Apdo. 210.

\section{Resumen}

Introducción. La planta de maracuyá es importante como fruta fresca y en la agroindustria, ya que es rica en minerales y vitaminas, además, posee potencial de consumo nacional y de exportación. Se ha demostrado que el nitrógeno $(\mathrm{N})$ tiene una influencia en el rendimiento de la fruta, pero no hay información sobre el rango óptimo de $\mathrm{N}$ para la producción máxima. Objetivo. Determinar la respuesta agronómica, fisiológica y fenológica, rendimiento y calidad de fruta en el cultivo de parchita maracuyá frente a diferentes dosis de nitrógeno. Materiales y métodos. En la Universidad Central de Venezuela, Maracay, Venezuela desde abril 2014 hasta marzo 2015 se sembraron plantas de maracuyá en un diseño completamente aleatorizado, seis repeticiones y seis plantas por unidad experimental, se evaluó un testigo (8 $\mathrm{g} \mathrm{N}_{\text { planta }}{ }^{-1}$ (T1)) y tres dosis de N (100 (T2), 200 (T3) y 300 (T4) g planta-1) y una dosis de 50 y 300 g planta ${ }^{-1}$ de $\mathrm{P}_{2} \mathrm{O}_{5}$ y $\mathrm{K}_{2} \mathrm{O}$, respectivamente. Las variables evaluadas fueron: altura de planta, número de hojas, tasa de fotosíntesis (A), transpiración (E), conductancia estomática (Gs), índice de clorofila (IC), contenido de $\mathrm{N}$ total en hoja en floración y fructificación, rendimiento y calidad de fruta. Resultados. A mayores dosis de N se obtuvieron mayores valores en altura, número de hojas, precocidad de planta, índice de clorofila, fotosíntesis neta y contenido de $\mathrm{N}$ foliar, y los menores valores en el T1. Las variables Gs, A y E, no presentaron diferencias significativas entre los tratamientos. Se obtuvo una reducción del rendimiento del $55 \%$ en el tratamiento testigo, comparado con el tratamiento de $200 \mathrm{~g}$ de $\mathrm{N}_{\text {planta }}{ }^{-1}$, el cual obtuvo el mayor rendimiento. El peso promedio de fruto fue significativamente mayor en T3. Conclusión. La mejor dosis nitrogenada en plantas de maracuyá fue la de $200 \mathrm{~g}$ de $\mathrm{N}_{\text {planta }}{ }^{-1}$.

Palabras clave: Passiflora edulis, nitrógeno, fisiología, rendimiento y calidad. 


\begin{abstract}
Introduction. The passion fruit plant is important as fresh fruit and in the agroindustry, since its rich in minerals and vitamins and also has potential for domestic consumption and exportation. It has been shown that nitrogen $(\mathrm{N})$ has an influence on fruit yield but there is no information on the optimal $\mathrm{N}$ range for maximum production. Objective. Determine the agronomic, physiological, and phenological response, yield and quality of fruit in the cultivation of parchita passion fruit against different doses of nitrogen. Materials and methods. At the Universidad Central de Venezuela, Maracay, Venezuela from April 2014 to march 2015, passion fruit plants were planted in a completely randomized design, six replicates, and six plants per experimental unit, a control $\left(8 \mathrm{~g} \mathrm{~N}\right.$ plant $\left.^{-1}(\mathrm{~T} 1)\right)$ was evaluated

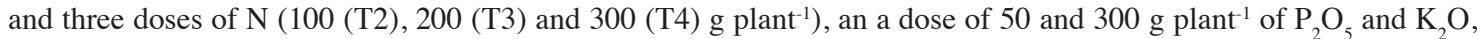
respectively were evaluated. The evaluated variables were: plant height, number of leaves, photosynthesis rate (A), transpiration (E), stomach conductance (Gs), chlorophyll index (CI), total leaf $\mathrm{N}$ content in doses at flowering and fruiting, yield, and fruit quality. Results. Higher doses of $\mathrm{N}$ obtained higher values in height, number of leaves, plant precocity, chlorophyll index, net photosynthesis, and foliar $\mathrm{N}$ content and lower values in T1. The variables Gs, A and E, did not show significant differences between the treatments. A $55 \%$ yield reduction was obtained in the control treatment compared to the $200 \mathrm{~g} \mathrm{~N}$ plant $^{-1}$ treatment which obtained the highest yield. The average fruit weight was significantly higher in T3. Conclusion. The best nitrogen dose in passion fruit plants was $200 \mathrm{~g} \mathrm{~N}$ plant ${ }^{-1}$.
\end{abstract}

Keywords: Passiflora edulis, nitrogen, physiology, yield and quality.

\title{
Introducción
}

El nitrógeno es uno de los elementos más importantes en la nutrición del cultivo de maracuyá (Passiflora edulis). Al respecto, Borges et al. (2006) indicaron que el $\mathrm{N}$ tiene una función estructural, como parte de moléculas de aminoácidos y proteínas, además de ser un constituyente de bases nitrogenadas y ácidos nucleicos. Actúa en procesos tales como absorción iónica, fotosíntesis, respiración, multiplicación, diferenciación de células y fundamentalmente en el crecimiento, formación vegetativa de la planta y la producción, ya que estimula el desarrollo de yemas florales y fructíferas, aumentando también el contenido de proteína. Por lo tanto, en ausencia de este nutriente, el crecimiento se retrasa, el tamaño de la planta es reducido y desarrolla ramas delgadas.

En el cultivo de maracuyá, las deficiencias de $\mathrm{N}$ se manifiestan como una clorosis moteada en hojas inferiores, con un posterior amarillamiento y secado, defoliación prematura y clorosis de otras hojas en forma ascendente, poco crecimiento de tallos y coloración rojiza de zarcillos con secado terminal (Guerrero et al., 2011).

El uso excesivo de fertilizantes nitrogenados está asociado a problemas de incremento en los costos de producción y de contaminación ambiental, por lo que se hace necesario contar con información que permita identificar las cantidades adecuadas para alcanzar los máximos rendimientos. Los modelos de absorción de $\mathrm{N}$ de los cultivos en función del clima y los métodos de diagnóstico de su estado en la planta, aparecen como una opción para hacer un uso más eficiente de los fertilizantes nitrogenados. En ese sentido, Borges et al. (2006) encontraron una disminución de la productividad con la aplicación de dosis más altas de nitrógeno $(0,100,200,400$ y $800 \mathrm{~kg}$ ha $\left.^{-1}\right)$, posiblemente atribuidas a la función del crecimiento vegetativo, resultando en una alta producción de hojas, y reducción del número de flores y, por consiguiente, una menor producción de frutos.

Las plantas cultivadas con cantidades inadecuadas de $\mathrm{N}$ normalmente no expresan su potencial productivo. En estas condiciones puede haber reducciones significativas en la relación de asimilación $\mathrm{CO}_{2}$, ya que el $\mathrm{N}$ es uno de los principales componentes del sistema fotosintético en la clorofila, en las enzimas ribulosa 1,5 bifosfato carboxilasa/oxigenasa (RUBISCO) y fosfoenolpiruvato carboxilasa (Coelho et al., 2010; Romero, 2012). 
En cuanto a la fisiología del cultivo, se señalan valores referenciales de plantas de gulupa (Passiflora edulis Sims) en las fases de floración y fructificación en condiciones normales de desarrollo, en el caso de transpiración,

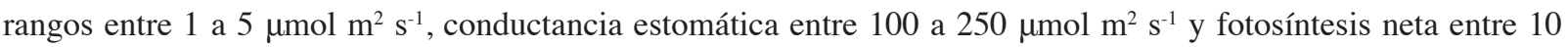
a $20 \mu \mathrm{mol} \mathrm{m} \mathrm{m}^{-1}$ (Pérez y Melgarejo, 2015). Esto se debe a que el intercambio de gases se lleva a cabo a través de los estomas y responde a diversos factores ambientales, entre los que destacan la luz, concentración de $\mathrm{CO}_{2} \mathrm{y}$ disponibilidad de nitrógeno (Schulze et al., 2005). Los asimilados, producidos por la fotosíntesis en los órganos fuente pueden ser almacenados o transportados, vía floema, a los diferentes órganos sumideros. Se considera que el suministro de nitrógeno a la planta traería consigo un aumento de su tasa fotosintética, incrementando el crecimiento de los cultivos dado que toda la materia seca producida por la planta depende totalmente de este proceso (Peil y Galvez, 2012).

En Brasil, con tecnologías de manejo para el cultivo de maracuyá han logrado obtener rendimientos entre 10 y $15 \mathrm{t} \mathrm{ha}^{-1}$ (Badiz et al., 2011). También se ha reportado que el potencial productivo podría llegar hasta $40 \mathrm{o} 50 \mathrm{t} \mathrm{ha}^{-1}$ (García et al., 2013). Los mejores rendimientos por hectárea se han informado cuando las densidades de siembra fueron 667 y 833 plantas; mientras que el nitrato de potasio produjo frutos de menor peso (Álvarez et al., 2018).

El objetivo de este trabajo fue determinar la respuesta agronómica, fisiológica y fenológica, rendimiento y calidad de fruta en el cultivo de parchita maracuyá frente a diferentes dosis de nitrógeno.

\section{Materiales y métodos}

Desde abril del 2014 hasta marzo del 2015, se estableció en el campo experimental del Instituto de Agronomía de la Facultad de Agronomía de la Universidad Central de Venezuela, Maracay, Venezuela, ubicada a 10 17'03" LN y 67³6'16" LO, a 455 msnm, para evaluar el efecto de diferentes dosis de nitrógeno sobre el desarrollo y la calidad de fruta en parchita maracuyá (Passiflora edulis), variedad maracuyá amarilla ‘región central'. El diseño del experimento fue en bloques al azar con cuatro tratamientos y seis repeticiones, para un total de veinticuatro unidades experimentales y seis plantas por unidad experimental (UE).

Se sembraron semillas en bandejas de germinación en etapa de vivero, al cumplir con el desarrollo óptimo de las plántulas, se trasplantaron en campo con un sistema de espaldera tipo cortina, con una sola línea de alambre a 1,80 m de altura. Se estableció una densidad de siembra equivalente a 1666 plantas ha $^{-1}$, mediante el arreglo de $3 \mathrm{~m}$ entre espalderas y $2 \mathrm{~m}$ entre plantas. Se utilizó un sistema de riego por goteo para la dotación hídrica del cultivo, la cual fue homogénea en toda el área experimental y estuvo basada de acuerdo con la evapotranspiración de referencia (Eto) registrada con un atmómetro, ajustando el Kc de acuerdo a la etapa fenológica del cultivo (Rodríguez, 2016).

Los tratamientos evaluados fueron dosis de 100, 200, $300 \mathrm{~g}$ de N planta $^{-1}$ (T2, T3 y T4, respectivamente) y el testigo $\mathrm{T} 1$ que recibió una dosis de $\mathrm{N}$ de $8 \mathrm{~g}$ de $\mathrm{N}_{\text {planta }}{ }^{-1}$. Debido a la fuente de fósforo utilizada, no hubo un testigo con $0 \mathrm{~g}$ de N planta ${ }^{-1}$. Las dosis de $\mathrm{P}$ y K fueron fijas para todas las plantas evaluadas (50 g planta ${ }^{-1}$ de $_{2} \mathrm{O}_{5}$ y $300 \mathrm{~g}$ planta $^{-1}$ de $\mathrm{K}_{2} \mathrm{O}$ ), como fuente de fósforo se usó fosfato diamónico (16-42-0) y para potasio el cloruro de potasio (0-0-50). Además, se utilizó como fuente de nitrógeno urea (46-0-0). Las dosis de nitrógeno se definieron de acuerdo con resultados previos de investigación obtenidos en el área experimental y validados en función a la densidad de plantas establecida del cultivo (Rodríguez, 2016).

Las fertilizaciones se fraccionaron de acuerdo a la etapa fenológica del cultivo (Maciel et al., 1994), en función del porcentaje del total requerido de la siguiente manera: $15 \%$ en la fase de crecimiento apical; $15 \%$ en la fase de crecimiento lateral; $25 \%$ en la fase de floración; $30 \%$ en la fase de fructificación y $15 \%$ en la fase de reposo vegetativo (Rodríguez, 2016).

Las variables evaluadas fueron las siguientes: 
- Altura de plantas y número de hojas: se evaluó semanalmente ambas variables en cuatro plantas por unidad experimental (plantas centrales de la parcela descartando extremos, para evitar el efecto de borde), hasta el momento en que llegaron a la altura del alambre en la espaldera.

- Fenología del cultivo: se llevó un registro del tiempo (días) de cada fase fenológica.

- Crecimiento apical: tiempo comprendido desde el transplante hasta que la planta alcanza la altura de 1,80 m.

- Crecimiento lateral: fase comprendida desde la ruptura de dominancia apical al llegar la planta al alambre, emisión de ramas laterales hasta el momento de la aparición de la primera flor.

- Floración: definida cuando el $50 \%$ de la población de cada unidad experimental tenga un botón floral desarrollado hasta la aparición del primer fruto.

- Fructificación: periodo comprendido desde la aparición del primer fruto hasta el final de cosecha.

- Contenido de nitrógeno total en tejido foliar: se seleccionaron cuatro hojas UE-1 con base en la cuarta hoja, en sentido ápice-base en ramas con botón floral, en etapa de floración y al final de la cosecha. Las muestras se secaron en estufa a $60{ }^{\circ} \mathrm{C}$ durante $48 \mathrm{~h}$. Se utilizó el método colorimétrico con el reactivo de Nessler descrito por Paneque et al. (2010).

- Índice de clorofila o grado de verdor: en la hoja indicadora (cuarta hoja sentido ápice-base) se tomaron dos hojas por planta y dos plantas por UE, con una frecuencia mensual desde la fase de crecimiento lateral de las ramas hasta final de cosecha. Se determinó con un equipo Spad Minolta 502, el cual determina la cantidad relativa de clorofila presente, mediante la medición de la absorción de la hoja en dos regiones de longitud de onda, roja y cercana a infrarroja; utilizando estas dos transmisiones, el medidor calcula el valor numérico Spad que es proporcional a la cantidad de clorofila presente en la hoja.

- Conductancia estomática (Gs; $\left.\mu \mathrm{mol} \mathrm{m} \mathrm{m}^{-2} \mathrm{~s}^{-1}\right)$, fotosíntesis $\left(\mathrm{A} ; \mu \mathrm{mol} \mathrm{m}^{-2} \mathrm{~s}^{-1}\right)$ y transpiración $\left(\mathrm{E} ; \mu \mathrm{mol} \mathrm{m}^{-2} \mathrm{~s}^{-1}\right)$ : esto se realizó en dos plantas por UE y dos hojas por planta entre las 9:00 y 11:00 a.m. con un medidor infrarrojo de gases (IRGA) CI-340 Photosynthesis Systems (CID Inc, Washington, USA), este equipo mide simultáneamente el intercambio de gases y tasa neta de fotosíntesis sobre la misma área foliar, con un control total sobre las variables ambientales.

- Rendimiento: se llevó un registro durante 51 semanas de cosecha en cada UE, los frutos maduros se cosecharon diariamente. Se midieron las variables producción por planta $\left(\mathrm{kg} \mathrm{planta}^{-1}\right)$ y su productividad equivalente a rendimiento $\left(\mathrm{t} \mathrm{ha}^{-1}\right)$, con base en la extrapolación de las mediciones del rendimiento por UE.

- Calidad de fruta: se determinaron variables físicas como: peso promedio del fruto (g), diámetro ecuatorial $(\mathrm{cm})$; largo del fruto $(\mathrm{cm})$, relación largo/ancho, grosor de cáscara $(\mathrm{mm}) ; \%$ de pulpa, \% de cascara y \% de semilla. En cuanto a las variables químicas, se determinó: $\mathrm{pH}$ (con un pH-metro portátil), sólidos solubles totales (SST) (con uso de un refractómetro marca ATC modelo Rbh, el cual basa su funcionamiento en el estudio de la refracción de la luz), acidez titulable (mediante la titulación con $\mathrm{NaOH} 0,1 \mathrm{~N}$ ), y relación SST/ acidez (se tomaron tres frutos maduros por UE en pleno periodo de cosecha provenientes de las seis plantas de la unidad experimental).

Los datos se analizaron por medio del paquete estadístico SAS versión 8.0 de acuerdo con el diseño propuesto. Se realizó un análisis de la varianza (ANAVAR) para cada variable y una prueba de medias de Waller-Duncan con un nivel de significancia $\alpha=0,05$.

\section{Resultados}

\section{Altura de la planta y número de hojas}

Los valores promedio de altura y número de hojas se tomaron hasta los setenta días después de la siembra, fecha en la cual el $50 \%$ de las plantas habían alcanzado la altura de 1,80 m, lo que se consideró como finalización de 
la fase de crecimiento apical (Cuadro 1). En ambas variables existieron diferencias estadísticamente significativas $(\mathrm{p} \leq 0,05)$ para los tratamientos evaluados.

Cuadro 1. Altura y número de hojas en el cultivo de maracuyá (Passiflora edulis), con la adición de nitrógeno. Maracay, Venezuela. Abril - mayo 2014.

Table 1. Height and number of leaves in the cultivation of passion fruit (Passiflora edulis), with nitrogen addition. Maracay, Venezuela. April - May 2014.

\begin{tabular}{ccc}
\hline Tratamientos & Altura de planta $(\mathbf{c m})$ & Número de hojas \\
\hline T1 (Testigo) & $146,04 \mathrm{c}$ & $15,70 \mathrm{c}$ \\
T2 & $149,56 \mathrm{c}$ & $16,50 \mathrm{c}$ \\
T3 & $176,54 \mathrm{a}$ & $19,75 \mathrm{a}$ \\
T4 & $168,90 \mathrm{~b}$ & $17,37 \mathrm{~b}$ \\
CV $(\%)$ & 9,31 & 4,98 \\
\hline
\end{tabular}

T1: 8 g N planta $^{-1}$; T2: $100 \mathrm{~g} \mathrm{~N}$ planta $^{-1}$; T3: $200 \mathrm{~g} \mathrm{~N}$ planta $^{-1}$; T4: $300 \mathrm{~g} \mathrm{~N}$ planta ${ }^{-1}$. CV: coeficiente de variación. Letras diferentes dentro de una misma columna se corresponden con diferencias estadísticamente significativas de acuerdo con la prueba de Waller-Duncan $\alpha=0,05$ / T1: $8 \mathrm{~g} \mathrm{~N}$ plant $^{-1}$; T2: $100 \mathrm{~g} \mathrm{~N}$ plant $^{-1}$; T3: $200 \mathrm{~g} \mathrm{~N}$ plant $^{-1}$; T4: $300 \mathrm{~g} \mathrm{~N}$ plant $^{-1}$. CV: variation coefficient. Different letters within the same column correspond to statistically significant differences according to the Waller-Duncan test $\alpha=0.05$.

Los mayores valores en ambas variables (altura de planta y número de hojas) se encontraron con la dosis de $200 \mathrm{~g}$ de $\mathrm{N}_{\text {planta-1 }}$ (T3), que superó a los encontrados con las dosis más elevada de N (T4).

\section{Fenología del cultivo}

Se observaron diferencias significativas entre los tratamientos estudiados para cada una de las fases consideradas. En el testigo existió un retraso en el desarrollo vegetativo, dada la mayor cantidad de días transcurridos en cada fase (Cuadro 2). Por el contrario, los tratamientos T3 y T4 presentaron mayor precocidad, lo cual indica el efecto favorable de la mayor dosis de nitrógeno sobre el desarrollo vegetativo de la planta. Del mismo modo, al observar

Cuadro 2. Duración de las fases fenológicas de crecimiento vegetativo con diferentes dosis de N en plantas de maracuyá (Passiflora edulis). Maracay, Venezuela. Abril 2014 - marzo 2015.

Table 2. Duration of the phenological phases of vegetative growth with different $\mathrm{N}$ doses in passion fruit (Passiflora edulis) plants. Maracay, Venezuela. April 2014 - March 2015.

\begin{tabular}{ccccc}
\hline Tratamientos & Crec. Apical & Crec. Lateral & Floración & Fructificación \\
\hline T1 (Testigo) & $55,25 \mathrm{a}$ & $26,75 \mathrm{a}$ & $76,93 \mathrm{a}$ & $198,07 \mathrm{~b}$ \\
T2 & $46,90 \mathrm{~b}$ & $21,68 \mathrm{~b}$ & $73,65 \mathrm{a}$ & $214,77 \mathrm{a}$ \\
T3 & $46,88 \mathrm{~b}$ & $19,16 \mathrm{~b}$ & $65,18 \mathrm{~b}$ & $225,78 \mathrm{a}$ \\
T4 & $46,70 \mathrm{~b}$ & $18,30 \mathrm{~b}$ & $66,86 \mathrm{~b}$ & $225,17 \mathrm{a}$ \\
CV $(\%)$ & 7,03 & 6,92 & 8,77 & 11,33 \\
\hline
\end{tabular}

T1: 8 g N planta $^{-1}$; T2: $100 \mathrm{~g} \mathrm{~N}$ planta $^{-1}$; T3: $200 \mathrm{~g} \mathrm{~N}$ planta $^{-1}$; T4: $300 \mathrm{~g} \mathrm{~N}$ planta ${ }^{-1}$. CV: coeficiente de variación. Letras diferentes dentro de una misma columna se corresponden con diferencias estadísticamente significativas de acuerdo con la prueba de Waller-Duncan $\alpha=0,05$ / T1: $8 \mathrm{~g} \mathrm{~N}$ plant $^{-1}$; T2: $100 \mathrm{~g} \mathrm{~N}$ plant $^{-1}$; T3: $200 \mathrm{~g} \mathrm{~N}$ plant $^{-1}$; T4: $300 \mathrm{~g} \mathrm{~N}$ plant $^{-1}$. CV: variation coefficient. Different letters within the same column correspond to statistically significant differences according to the Waller-Duncan test $\alpha=0.05$. 
la fase de fructificación se vio que en el tratamiento testigo se redujo el periodo a la cosecha y presentó una menor cantidad de días con respecto al resto de los tratamientos estudiados.

\section{Variables fisiológicas}

Se observó que para las evaluaciones iniciales (de agosto a octubre) no se presentaron diferencias significativas entre tratamientos (Figura 1), no obstante, a medida que se avanzó en el ciclo del cultivo se hicieron más evidentes los menores valores de Spad en el testigo (T1), lo cual denota que el grado de verdor de las hojas fue menor, esto dado por la posible deficiencia de nitrógeno. En el resto de los tratamientos estudiados, los valores incrementaron conforme las dosis de nitrógeno también aumentaron. Las evaluaciones correspondientes a los meses entre noviembre a febrero presentaron diferencias estadísticamente significativas entre tratamientos evaluados. Los primeros meses de evaluación no permitieron obtener respuestas diferenciadas entre los niveles de fertilización nitrogenada, fue a partir de octubre que se manifestaron tendencias en el índice de clorofila, de acuerdo con los niveles de fertilización. En este sentido, los valores normales para el cultivo se encontraron en el rango de 40 a 50 unidades Spad (Rodríguez, 2016). De esta forma, el tratamiento testigo (T1) fue el que presentó una disminución en el contenido por debajo de los valores adecuados para el cultivo. Es necesario indicar que dichas evaluaciones se realizaron desde la fase de crecimiento lateral de las ramas hasta la fructificación (siete meses continuos de evaluación desde agosto 2014 hasta febrero 2015).

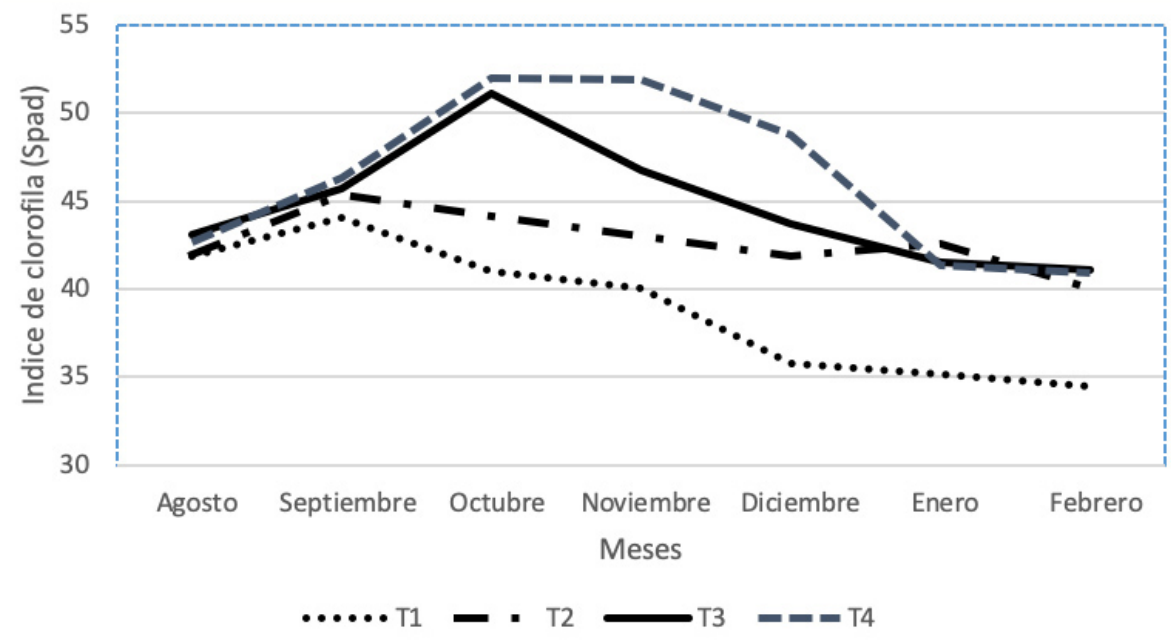

Figura 1. Índice de clorofila (unidades Spad) durante el desarrollo de plantas de maracuyá (Passiflora edulis) sometidas a diferentes dosis de $\mathrm{N}$ desde agosto 2014 hasta febrero 2015. Maracay, Venezuela.

T1: testigo 8 g N planta-1; T2: $100 \mathrm{~g} \mathrm{~N}$ planta $^{-1}$; T3: $200 \mathrm{~g} \mathrm{~N}$ planta-1 $^{-1}$ T4: $300 \mathrm{~g} \mathrm{~N}$ planta $^{-1}$.

Figure 1. Chlorophyll index (Spad units) during the development of passion fruit (Passiflora edulis) plants subjected to different $\mathrm{N}$ doses from August 2014 to February 2015, Maracay, Venezuela.

T1: 8 g N plant ${ }^{-1} ; \mathrm{T} 2: 100 \mathrm{~g} \mathrm{~N}$ plant $^{-1} ; \mathrm{T} 3: 200 \mathrm{~g} \mathrm{~N}$ plant $^{-1} ; \mathrm{T} 4: 300 \mathrm{~g} \mathrm{~N}$ plant $^{-1}$.

Referente a los efectos sobre las variables fisiológicas, los resultados se presentan en el Cuadro 3. En cuanto al intercambio gaseoso se refiere, no hubo diferencias estadísticas significativas para las variables transpiración (E) y conductancia estomática (Gs); mientras que en el caso de la fotosíntesis neta (A), se presentaron diferencias entre tratamientos para ambas fechas de evaluación. 
Cuadro 3. Valores de fotosíntesis en intercambio gaseoso $\left(\mu \mathrm{mol} \mathrm{m} \mathrm{m}^{-2} \mathrm{~s}^{-1}\right.$ ) en plantas de maracuyá (Passiflora edulis) bajo diferentes dosis de N, en la etapa de floración y fructificación. Maracay, Venezuela. Agosto 2014 - febrero 2015.

Table 3. Values of photosynthesis in gas exchange ( $\mu \mathrm{mol} \mathrm{m-2} \mathrm{s-1)} \mathrm{in} \mathrm{passion} \mathrm{fruit} \mathrm{(Passiflora} \mathrm{edulis)} \mathrm{plants} \mathrm{under} \mathrm{different} \mathrm{N}$ doses in the flowering and fruiting stage. Maracay, Venezuela. August 2014 - February 2015.

\begin{tabular}{|c|c|c|c|c|c|c|}
\hline \multirow{2}{*}{ Tratamiento } & A1 & $\mathbf{A 2}$ & Gs1 & Gs2 & E1 & $\mathbf{E 2}$ \\
\hline & \multicolumn{6}{|c|}{$\left(\mu \mathrm{mol} \mathrm{m} \mathrm{m}^{-2} \mathrm{~s}^{-1}\right)$} \\
\hline T1 (Testigo) & $11,18 \mathrm{c}$ & $10,15 \mathrm{c}$ & $164,48 \mathrm{a}$ & $150,30 \mathrm{a}$ & $1,48 \mathrm{a}$ & $1,64 \mathrm{a}$ \\
\hline $\mathbf{T} 2$ & $13,63 \mathrm{~b}$ & $12,00 \mathrm{~b}$ & $163,36 \mathrm{a}$ & $152,18 \mathrm{a}$ & $1,64 \mathrm{a}$ & $1,73 \mathrm{a}$ \\
\hline T3 & $14,15 \mathrm{~b}$ & $12,80 \mathrm{~b}$ & $203,64 \mathrm{a}$ & $160,41 \mathrm{a}$ & $1,80 \mathrm{a}$ & $1,89 \mathrm{a}$ \\
\hline T4 & $17,20 \mathrm{a}$ & $18,15 \mathrm{a}$ & 195,09 a & $168,98 \mathrm{a}$ & $1,65 \mathrm{a}$ & $1,87 \mathrm{a}$ \\
\hline $\mathrm{CV}(\%)$ & 8,31 & 9,20 & 8,36 & 10,58 & 4,36 & 6,58 \\
\hline
\end{tabular}

T1: $8 \mathrm{~g} \mathrm{~N}$ planta $^{-1}$; T2: $100 \mathrm{~g} \mathrm{~N}$ planta $^{-1}$; T3: $200 \mathrm{~g} \mathrm{~N}$ planta $^{-1}$; T4: $300 \mathrm{~g} \mathrm{~N}$ planta $^{-1}$. A: fotosíntesis neta; Gs: conductancia estomática; E: transpiración; 1: etapa de floración; 2: etapa de fructificación. CV: coeficiente de variación. Letras diferentes dentro de una misma columna se corresponden con diferencias estadísticamente significativas de acuerdo con la prueba de Waller-Duncan $\alpha=0,05$ / T1: $8 \mathrm{~g} \mathrm{~N}$ plant $^{-1}$; T2: $100 \mathrm{~g} \mathrm{~N}$ plant $^{-1}$; T3: $200 \mathrm{~g} \mathrm{~N}$ plant $^{-1}$; T4: $300 \mathrm{~g} \mathrm{~N}$ planta $^{1}$. A: net photosynthesis; Gs: stomatal conductance; E: transpiration; 1: flowering stage; 2: fruiting stage. CV: variation coefficient. Different letters within the same column correspond to statistically significant differences according to the Waller-Duncan test $\alpha=0.05$.

Los valores de contenido de nitrógeno al momento de floración y cosecha se presentan en el Cuadro 4.

Cuadro 4. Valores de nitrógeno en tejido foliar en plantas de maracuyá (Passiflora edulis), bajo diferentes dosis de N, en etapa de floración y final de cosecha. Maracay, Venezuela. Agosto 2014 - febrero, 2015.

Table 4. Nitrogen values in foliar tissue in passion fruit (Passiflora edulis) plants under different $\mathrm{N}$ doses in flowering stage and end of harvest. Maracay, Venezuela. August 2014 - February, 2015.

\begin{tabular}{ccc}
\hline Tratamientos & $\begin{array}{c}\text { Nitrógeno total }(\%) \\
\text { floración }\end{array}$ & $\begin{array}{c}\text { Nitrógeno total (\%) } \\
\text { final de cosecha }\end{array}$ \\
\hline T1 (Testigo) & $3,31 \mathrm{~d}$ & $2,09 \mathrm{c}$ \\
T2 & $3,94 \mathrm{c}$ & $2,60 \mathrm{~b}$ \\
T3 & $4,77 \mathrm{~b}$ & $2,69 \mathrm{~b}$ \\
T4 & $5,31 \mathrm{a}$ & $3,75 \mathrm{a}$ \\
CV $(\%)$ & 7,36 & 6,90 \\
\hline
\end{tabular}

T1: $8 \mathrm{~g} \mathrm{~N}$ planta $^{-1}$; T2: $100 \mathrm{~g} \mathrm{~N}$ planta $^{-1}$; T3: $200 \mathrm{~g} \mathrm{~N}$ planta $^{-1}$; T4: $300 \mathrm{~g} \mathrm{~N}$ planta ${ }^{-1}$. CV: coeficiente de variación. Letras diferentes dentro de una misma columna se corresponden con diferencias estadísticamente significativas de acuerdo con la prueba de Waller-Duncan $\alpha=0,05$ / T1: $8 \mathrm{~g} \mathrm{~N}$ plant $^{-1}$; T2: $100 \mathrm{~g} \mathrm{~N}$ plant $^{-1}$; T3: $200 \mathrm{~g} \mathrm{~N}$ plant $^{-1}$; T4: $300 \mathrm{~g} \mathrm{~N}$ plant $^{-1}$. CV: variation coefficient. Different letters within the same column correspond to statistically significant differences according to the Waller-Duncan test $\alpha=0.05$.

\section{Rendimiento y calidad de fruta}

En relación con el rendimiento evaluado durante un ciclo completo de producción (primer año del cultivo), los resultados se presentan en el Cuadro 5 . Al respecto, se observaron diferencias significativas entre tratamientos para las variables asociadas a la productividad del cultivo $\left(\mathrm{kg}_{\text {planta }}{ }^{-1} \mathrm{y} \mathrm{tha}^{-1}\right)$.

Las plantas con dosis de $200 \mathrm{~g} \mathrm{~N}$ planta $^{-1}$ (T3) presentaron el mayor rendimiento en comparación con el resto de los tratamientos, lo cual sugiere que la dosis más alta de nitrógeno evaluada no necesariamente implique mayor productividad. 
Cuadro 5. Rendimiento de plantas de maracuyá (Passiflora edulis), bajo diferentes dosis de nitrógeno. Maracay, Venezuela.Agosto 2014 - junio 2015.

Table 5. Yield of passion fruit (Passiflora edulis) plants under different doses of nitrogen. Maracay, Venezuela. August 2014-June 2015.

\begin{tabular}{ccc}
\hline Tratamientos & Rendimiento $\left(\mathbf{k g ~ p l a n t a}^{-1}\right)$ & Rendimiento $^{\left(\mathbf{t ~ h a}^{-1}\right)}$ \\
\hline T1 (Testigo) & $3,53 \mathrm{c}$ & $5,88 \mathrm{c}$ \\
T2 & $6,48 \mathrm{~b}$ & $10,79 \mathrm{~b}$ \\
T3 & $9,12 \mathrm{a}$ & $15,19 \mathrm{a}$ \\
T4 & $8,62 \mathrm{a}$ & $14,36 \mathrm{a}$ \\
CV $(\%)$ & 11,36 & 10,89 \\
\hline
\end{tabular}

T1: testigo sin N; T2: $100 \mathrm{~g} \mathrm{~N}$ planta $^{-1}$; T3: $200 \mathrm{~g} \mathrm{~N}$ planta $^{-1}$; T4: $300 \mathrm{~g} \mathrm{~N}$ planta ${ }^{-1}$. CV: coeficiente de variación. Letras diferentes dentro de una misma columna se corresponden con diferencias estadísticamente significativas de acuerdo la prueba de Waller-Duncan $\alpha=0,05$ / T1: $8 \mathrm{~g} \mathrm{~N}$ plant $^{-1}$; T2: $100 \mathrm{~g} \mathrm{~N}$ plant $^{-1}$; T3: $200 \mathrm{~g} \mathrm{~N}$ plant $^{-1}$; T4: $300 \mathrm{~g} \mathrm{~N} \mathrm{plant}^{-1}$. CV: variation coefficient. Different letters within the same column correspond to statistically significant differences according to the Waller-Duncan test $\alpha=0.05$.

En cuanto a la calidad de fruta, se evaluaron para los diferentes tratamientos variables físicas y químicas que son importantes tener en cuenta para la cuantificación de parámetros de calidad exigidos tanto en mercado de fruta fresca como procesamiento agroindustrial. Los resultados se muestran en el Cuadro 6.

Cuadro 6. Valores de calidad de fruta evaluados en plantas de maracuyá (Passiflora edulis), bajo diferentes dosis de N, en época de cosecha. Maracay, Venezuela. Marzo, 2015.

Table 6. Fruit quality values evaluated in passion fruit (Passiflora edulis) plants under different $\mathrm{N}$ doses at harvest time. Maracay, Venezuela. March, 2015.

\begin{tabular}{|c|c|c|c|c|c|c|c|c|c|c|}
\hline Trat. & $\begin{array}{c}\text { Peso } \\
\text { fruto }(g)\end{array}$ & Rel. L/A & $\begin{array}{c}\text { Grosor } \\
\text { cáscara }(\mathbf{m m})\end{array}$ & $\begin{array}{c}\% \\
\text { cáscara }\end{array}$ & $\begin{array}{c}\% \\
\text { semilla }\end{array}$ & $\%$ jugo & SST (\%) & $\begin{array}{l}\text { Acidez } \\
\text { (A) }\end{array}$ & $\begin{array}{c}\text { Rel. } \\
\text { SST/A }\end{array}$ & pH \\
\hline T1 & $168,29 \mathrm{~b}$ & 1,16 & 5,72 & 42,66 & 13,20 & 46,66 & 14,12 & 3,78 & 3,77 & 3,40 \\
\hline $\mathbf{T} 2$ & $174,13 \mathrm{~b}$ & 1,18 & 5,80 & 42,33 & 12,35 & 44,48 & 14,61 & 3,78 & 3,92 & 3,37 \\
\hline T3 & $203,31 \mathrm{a}$ & 1,14 & 5,91 & 42,03 & 11,91 & 45,61 & 15,00 & 3,65 & 4,12 & 3,65 \\
\hline T4 & $183,42 \mathrm{ab}$ & 1,22 & 5,68 & 41,81 & 13,03 & 42,51 & 14,60 & 3,84 & 3,83 & 3,84 \\
\hline CV $(\%)$ & 59,16 & 1,18 & 6,43 & 26,51 & 7,81 & 27,92 & 15,74 & 3,32 & 4,81 & 3,33 \\
\hline
\end{tabular}

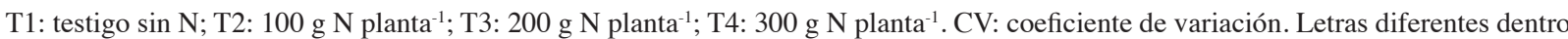
de una misma columna se corresponden con diferencias estadísticamente significativas de acuerdo la prueba de Waller-Duncan $\alpha=0,05$ / T1: $8 \mathrm{~g} \mathrm{~N}$ plant $^{-1}$; T2: $100 \mathrm{~g} \mathrm{~N}$ plant $^{-1}$; T3: $200 \mathrm{~g} \mathrm{~N}$ plant $^{-1}$; T4: $300 \mathrm{~g} \mathrm{~N} \mathrm{plant}^{-1}$. CV: variation coefficient. Different letters within the same column correspond to statistically significant differences according to the Waller-Duncan test $\alpha=0.05$.

\section{Discusión}

En el cultivo de maracuyá, Gusqui et al. (2009) señalaron que diferentes dosis de fertilización con N-P-K, no influyeron en la altura de las plantas con el uso de fórmula completa 10-30-10; asimismo, hicieron referencia que, de acuerdo con el análisis estadístico, las dosis de fertilizante no influyeron en el número de hojas durante la fase de desarrollo vegetativo, esto sugiere que no necesariamente un aumento en la dosis de $\mathrm{N}$ sea favorable exponencialmente para el desarrollo del cultivo. Este comportamiento fue contrario al de esta investigación, ya 
que en este caso la evaluación fue hasta que la etapa fenológica de crecimiento apical, lo que dio indicios que la aplicación de nitrógeno tuvo un efecto diferencial y acumulativo a lo largo de las diferentes etapas fenológicas.

En un ensayo sobre fertilización del cultivo de maracuyá con fuentes N-P-K en Ecuador, se encontró que el número de días transcurridos desde el trasplante hasta la cosecha con uso de dosis de 100-50-80 $\mathrm{kg} \mathrm{ha}^{-1} \mathrm{en}_{\text {forma }}$ bimensual, se redujo en comparación al resto de los tratamientos evaluados; adicionalmente, dicho tratamiento duró 186 días, para iniciar la cosecha, mientras que en el testigo inició a los 210 días (Pereira, 2015). Los resultados encontrados en la presente investigación mostraron mayor precocidad para el inicio de la etapa productiva del cultivo bajo las condiciones de experimentación, Fischer et al. $(2010 ; 2016)$ señalaron que esto podría deberse a que en regiones más cercanas al Ecuador, el inicio de la fructificación tiende a ser más precoz.

El Spad permite conocer el contenido de nitrógeno de la planta, esto debido a que existe una correlación significativa entre el grado de verdor y la concentración de clorofila y nitrógeno foliar. Plantas de maracuyá sometidas a estrés hídrico presentaron valores entre 30 y 40 unidades Spad, con clorosis de las hojas (Gaudio, 2011). Por su parte, Gaudio (2011) indicó valores normales entre 40 y 50 unidades Spad para este cultivo.

La tendencia general, indistintamente del tratamiento considerado, es a la disminución del IC, dado por la movilización de $\mathrm{N}$ hacia los tejidos reproductivos (flores y frutos), lo cual se observó en las evaluaciones a partir de diciembre. Resultados similares han sido señalados por Rodríguez (2016) en el cultivo de maracuyá en la misma área experimental de la presente investigación.

Los resultados de la presente investigación demostraron que los mayores valores de fotosíntesis neta se encontraron en el tratamiento T3, y disminuyeron progresivamente mientras menor fue la dosis aplicada de $\mathrm{N}$ en las plantas, con los menores valores en el tratamiento testigo $\left(8 \mathrm{~g} \mathrm{~N} \mathrm{planta}^{-1}\right)$.

La determinación del contenido de nitrógeno total en tejido foliar fue realizado en floración y al final de cosecha, destacando que en la primera fase los valores fueron mayores y disminuyeron en la segunda. Esto es debido a la relación fuente - sumidero, donde el contenido de nitrógeno se moviliza hacia las flores y frutos una vez comenzados estos procesos en la etapa reproductiva de la planta, siendo el $\mathrm{N}$ el segundo elemento mayormente exportado hacia el fruto después del K (Rodríguez, 2016). Resultados similares de disminución en el contenido de nitrógeno desde la floración hasta la fructificación han sido reportados en el cultivo de maracuyá, con base en la determinación de nitrato en savia y nitrógeno total en tejido foliar (Rodríguez, 2016).

Los valores óptimos de $\mathrm{N}$ en la hoja de maracuyá varían entre 3,6 y 4,6 \% (Gusqui et al., 2009). De esta forma, únicamente el testigo obtuvo valores inferiores de los niveles óptimos señalados anteriormente; mientras que los tratamientos T3 y T4 presentaron valores superiores, indicando así una adecuada nutrición de las plantas evaluadas en fase de floración. El mayor valor de contenido de nitrógeno foliar total correspondió al tratamiento al que se le aplicó la dosis máxima de nitrógeno, el cual es un comportamiento esperado, ya que las plantas de maracuyá que tienen más disponible el nutriente en el suelo son las que tienen la posibilidad de acumular más contenido del elemento en la hoja.

Se observó una disminución del contenido de $\mathrm{N}$ de la floración a la cosecha, esto es debido a la relación fuentesumidero explicado previamente.

Con relación al rendimiento, Borges et al. (2006) encontraron una disminución de la productividad con la aplicación de dosis más altas de nitrógeno $\left(0,100,200,400\right.$ y $800 \mathrm{~kg} \mathrm{~N} \mathrm{ha}^{-1}$ año $\left.^{-1}\right)$, posiblemente atribuidas a la función del crecimiento vegetativo, resultando en una alta producción de hojas y reduciendo el número de flores y, por consiguiente, menos cantidad de frutos. Las menores dosis de nitrógeno (T1 y T2) obtuvieron los menores rendimientos; esto tiene relación con que dicho nutrimento está fuertemente asociado al incremento en la productividad de los cultivos (Fischer et al., 2010).

De acuerdo con los resultados de rendimiento obtenidos en la presente investigación, las plantas con la menor aplicación de N (T1) obtuvieron una reducción del 61,25\% de la productividad con respecto al tratamiento de mayor producción (T3), lo cual pone de manifiesto la importancia de realizar una adecuada fertilización nitrogenada 
en el desarrollo y producción del cultivo. Es necesario destacar que el suelo bajo estudio presentó bajos valores de materia orgánica ( 0,93 y 0,86 para profundidades de 0-20 y 20 a $40 \mathrm{~cm}$, respectivamente). Por su parte, Álvarez et al. (2018) evaluaron tres densidades de siembra y tres fuentes nitrogenadas, entre ellas, urea $\left(\mathrm{CH}_{4} \mathrm{~N}_{2} \mathrm{O}\right)$, nitrato de calcio $\mathrm{Ca}\left(\mathrm{NO}_{3}\right)_{2}$, nitrato de potasio $\left(\mathrm{KNO}_{3}\right)$ y nitrato de amonio $\left(\mathrm{NH}_{4} \mathrm{NO}_{3}\right)$, el mejor peso de fruto $(252,60 \mathrm{~g})$ se obtuvo en la combinación de 555 plantas ha ${ }^{-1}$ más urea. En este mismo estudio, no se observaron diferencias significativas en los factores independientes de fuentes nitrogenadas con una dosis de $\mathrm{N}$ por planta de $1440 \mathrm{~g}$ por ciclo.

Dentro de los aspectos evaluados de calidad, el peso promedio del fruto fue la única variable que presentó diferencias estadísticamente significativas entre los tratamientos estudiados, tanto para los parámetros físicos como químicos de calidad. Se mantuvo la tendencia en T3 de presentar los mayores valores, seguido de T4, T2 y T1, respectivamente. No obstante, los valores de peso del fruto obtenidos, incluso en el testigo, fueron superiores a los reportados en otras investigaciones en el país (Aular y Casares, 2011; Pérez y Melgarejo, 2012) donde se han encontrado valores entre 100 y $130 \mathrm{~g}^{\text {fruto }^{-1}}$; lo cual indica que, con respecto al tamaño del fruto, no se produjeron resultados desfavorables en cuanto a calidad física se refiere. Por su parte, Álvarez et al. (2018) reportaron en su investigación que las fuentes nitrogenadas urea $\left(\mathrm{CH}_{4} \mathrm{~N}_{2} \mathrm{O}\right)$, nitrato de calcio $\mathrm{Ca}\left(\mathrm{NO}_{3}\right)_{2}$, nitrato de potasio $\left(\mathrm{KNO}_{3}\right)$ y nitrato de amonio $\left(\mathrm{NH}_{4} \mathrm{NO}_{3}\right)$, produjeron un grosor de cáscara de $5 \mathrm{~mm}$ y no hubo diferencias significativas en el porcentaje de la pulpa.

Desde el punto de vista de los criterios de calidad exigidos normalmente por la agroindustria nacional señalados por Roa (2011), ninguno de los tratamientos evaluados presentó resultados desfavorables en tales parámetros, por lo cual las dosis de nitrógeno estudiadas no incidieron notablemente sobre la calidad del producto cosechado. Los rangos óptimos exigidos por la agroindustria para las características químicas son valores de SST > 14; AT entre 2,5 y 3,$5 ; \mathrm{SST} / \mathrm{AT}>4$ y pH entre 2,8 y 4,0 (Roa, 2011). En esta investigación las plantas sometidas a los cuatro tratamientos de nutrición nitrogenada estuvieron en el rango óptimo para las variables asociadas a calidad de fruta.

Para las variables asociadas a calidad de fruta, Hafle et al. (2009) reportaron los siguientes resultados: 6,48 $\mathrm{mm}$ de grosor promedio de cáscara, $9,03 \mathrm{~mm}$ de diámetro longitudinal, 7,9 $\mathrm{mm}$ de diámetro ecuatorial, peso promedio de frutos de 193,10 g, porcentajes cáscaras de $62,43 \%, 32,42 \%$ de jugo y 5,22 \% de semilla. Entre las variables químicas que midieron se encontraron SST $(13,14 \%)$, AT $(5,02)$ y SST/AT $(2,62)$. Al comparar los valores encontrados en la presente investigación con los obtenidos por estos se puede determinar que los resultados del presente estudio fueron incluso más favorables dentro de los atributos de calidad para el cultivo, con mayor cantidad de SST, \% jugo y peso promedio del fruto.

Autores indicaron como dosis referencial, para la región noreste del Brasil, el uso de $205 \mathrm{~kg} \mathrm{ha}^{-1}$ para suplir las necesidades nutricionales del cultivo de maracuyá (Borges et al., 2006). En cuanto a calidad se refiere, Borges et al. (2006) encontraron una mayor cantidad de sólidos solubles totales y menor acidez en el jugo, así como el aumento de la productividad fue posible con la aplicación de $205 \mathrm{~kg} \mathrm{ha}^{-1}$ en el suelo, de esta manera, en términos generales, en la presente investigación no se presentaron efectos significativos sobre la calidad de la fruta con la aplicación de diferentes dosis de nitrógeno, con excepción de la variable peso promedio del fruto, y se mantuvieron los estándares adecuados en los parámetros considerados de acuerdo a las exigencias del sector agroindustrial nacional.

\section{Conclusiones}

La aplicación de dosis menores a $200 \mathrm{~g} \mathrm{~N}$ planta $^{-1}$ afectó negativamente el desarrollo de la planta, hubo menor altura y número de hojas, así como un retraso en el desarrollo del cultivo, disminución del contenido de $\mathrm{N}$, fotosíntesis y rendimiento del cultivo.

La dosis de nitrógeno que presentó mejor resultado en cuanto a desarrollo, fisiología, rendimiento y calidad de fruta fue $200 \mathrm{~g} \mathrm{~N}_{\text {planta }}^{-1}$, por lo cual se recomienda emplear dicha dosis en investigaciones posteriores, bajo condiciones similares a las de esta experimentación. 


\section{Agradecimientos}

Los autores agradecen al Fondo Nacional de Ciencia y Tecnología (FONACIT) por los recursos financieros para el desarrollo del proyecto $\mathrm{N}^{\mathrm{0}} 2013-002353$ "Ecofisiología del estrés en el cultivo de parchita maracuyá como alternativa tecnológica frente a las principales limitantes en zonas del centro del país" desarrollado en la Universidad Central de Venezuela, Facultad de Agronomía, Venezuela.

\section{Literatura citada}

Álvarez, H., J. Pionce, J. Castro, W. Viera, y A. Sotomayor. 2018. Densidades poblacionales y fertilización nitrogenada en maracuyá. Ecuador es Calidad: Rev. Cient. Ecuatoriana 5(1):1-6.

Aular, J., y M. Casares. 2011. Consideraciones sobre la producción de frutas en Venezuela. Rev. Bras. Frutic. 33(esp1):187-198. doi:10.1590/S0100-29452011000500022

Badiz, F., A. Martins, M. Esperancini, A. Vidal, e F. Okamoto. 2011. Custo de produção do maracujazeiro-amarelo (Passiflora edilus). Rev. Bras. Frutic. 33:441-446. doi:10.1590/S0100-29452011000500058

Borges, A., R. Correa, e A. Almeida. 2006. Doses e fontes de nitrogênio em fertirrigação no cultivo do maracujá-amarelo. Rev. Bras. Frutic. 28:301-314. doi:10.1590/S0100-29452006000200033

Coelho, F., P.C. Fontes, M. Puiatti, J.C. Neves y M.C. Silva. 2010. Dose de nitrogênio associada à produtividade de batata e índices do estado de nitrogênio na folha. Rev. Bras. Ciênc. Solo 34:1175-1183. doi:10.1590/S0100-06832010000400017

Fischer, I., A. Marques, M. Fileti, R. Marques, M. De-Arrula, e C. Bueno. 2010. Avaliação de passifloras, fungicidas e trichoderma para o manejo da podridão-do-colo do maracujazeiro causada por Nectria haematococca. Rev. Bras. Frutic. 32:709-717. doi:10.1590/S0100-29452010005000090

Fischer, G., F. Ramírez, and F. Casierra-Posada. 2016. Ecophysiological aspects of fruit crops in the era of climate change. A review. Agron. Colomb. 34(2):190-199. doi:10.15446/agron.colomb.v34n2.56799

García, C., O. Nunes, C. Da-Silva, e J. De-Oliveira. 2013. AvaliaÇão agronômica de parentais e híbridos de maracujazeiroamarelo. Rev. Bras. Frutic. 35:191-198. doi:10.1590/S0100-29452013000100022

Gaudio, M. 2011. Respostas ecofisiológicas e bioquimicas do maracujazeiro (Passiflora edulis Sims) ao déficit hídrico. Tesis M.Sc., Universidade Federal do Espiritu Santo, Vitória, BRA.

Guerrero, E., C. Potosí, L. Melgarejo, y L. Hoyos. 2011. Manejo agronómico de la gulupa (Passiflora edulis Sims) en el marco de las buenas prácticas agrícolas. En: L. Melgarejo, editor, Ecofisiología del cultivo de la gulupa (Passiflora edulis Sims). Universidad Nacional de Colombia, Bogotá, COL. p. 123-144.

Gusqui, L.W., M.N. Recalde, X. López, y J. Jumbo. 2009. Determinación de curvas de absorción de macroelementos, durante el primer año de desarrollo del cultivo de maracuyá (Passiflora edulis) en Santo Domingo de los de los Tsáchilas, Ecuador, Diciembre 2018. Tsafiqui. 1:12-36. doi:10.29019/tsafiqui.v0i1.10

Hafle, O., J. Ramos, L. De-Oliveira, E. Ferreira, e P. De-Melo. 2009. Produtividade e qualidade de frutos do maracujazeiroamarelo Submetido a poda e ramos productivos. Rev. Bras. Frutic. 31:736-770. doi:10.1590/S0100-29452009000300020

Maciel, N., D. Bautista, y J. Aular. 1994. Crecimiento, desarrollo y arquitectura de Passiflora edulis f. flavicarpa. Proc. Interamer. Soc. Trop. Hort. 38:133-138.

Paneque, V.M., J. Calaña, M. Calderón, Y. Borges, T. Hernández, y M. Caruncho. 2010. Manual de técnicas analíticas para análisis de suelos, foliar, abonos orgánicos y fertilizantes químicos. Instituto Nacional de Ciencias agrícolas (INCA), La Habana, CUB. 
Peil, R.M., y J. Galvez. 2012. Reparto de materia seca como factor determinante de la producción de las hortalizas de fruto cultivadas en invernadero. Rev. Bras. Agrociênc. 11:5-11. doi:10.18539/cast.v11i1.1171

Pereira, V. 2015. Estudio a la aplicación de tres frecuencias y dos dosis de N-P-K más una fórmula de fertilizante foliar en el cultivo de maracuyá. Tesis Lic., Universidad de Guayaquil, Guayaquil, ECU.

Pérez, L., y M. Melgarejo. 2012. Caracterización ecofisiológica de gulupa (Passiflora edulis Sims) bajo tres condiciones ambientales del departamento de Cundinamarca. En: M. Melgarejo, editor, Ecofisiología del cultivo de gulupa (Passiflora edulis Sims). Universidad Nacional de Colombia, Bogotá, COL. p. 11-32.

Pérez, L., y M. Melgarejo. 2015. Photosynthetic performance and leaf water potential of gulupa (Passiflora edulis Sims) in the reproductive phase in three locations in the Colombian Andes. Acta Biol. Colomb. 20:183-194. doi:10.15446/abc. v20n1.42196

Roa, D. 2011. Exigencias del mercado de la parchita maracuyá (Passiflora edulis Sims f. flavicarpa Degener) en los estados Aragua y Carabobo. Tesis Lic., Universidad Central de Venezuela, Caracas, VEN.

Romero, J. 2012. Análisis de la dinámica hídrica de la planta tipo C3, caso de estudio: cultivo de maracuyá (Passiflora edulis variedad flavicarpa) en condiciones de clima templado. Tesis M.Sc., Pontificia Universidad Javeriana, Bogotá, COL.

Rodríguez, G. 2016. Evaluación del manejo de parchita maracuyá (Passiflora edulis Sims) en ciclo anual, con riego deficitario controlado y control biológico de Fusarium spp. a base de Trichoderma spp. Tesis Ph.D., Universidad Central de Venezuela, Caracas, VEN.

Schulze, E., E. Beck, and K. Müller. 2005. Plant ecology. Springer Verlag, Berlin, GER. 